\title{
Improvement of poly(3-hexylthiophene-2,5-diyl) electron mobility through complete elimination of regioregularity defects
}

\author{
Ali Nawaz ${ }^{\mathrm{a}, b^{*}}$, Marlus Koehler ${ }^{\mathrm{a}}$, Anil Kumar ${ }^{\mathrm{c}}$, Leandro Merces ${ }^{\mathrm{d}}$, Anne B. R. Abreu ${ }^{\mathrm{a}}$, Carlos C. B. Bufon ${ }^{\mathrm{e}}$, \\ Jose P. M. Serbena ${ }^{\text {a }}$ Ivo A. Hümmelgen ${ }^{\mathrm{a}, \uparrow}$ \\ ${ }^{a}$ Departamento de Física, Universidade Federal do Paraná, Caixa Postal 19044, 81531-980 Curitiba, PR, \\ Brazil \\ ${ }^{b}$ Center for Sensors and Devices, Bruno Kessler Foundation (FBK), 38123, Trento, Italy \\ ${ }^{c}$ Indian Institute of Technology, Bombay, Powai Mumbai 400076, India
}

${ }^{d}$ Brazilian Nanotechnology National Laboratory (LNNano), Brazilian Center for Research in Energy and Materials (CNPEM), 13083-970 Campinas, São Paulo, Brazil

${ }^{e}$ MackGraphe - Graphene and Nanomaterials Research Center, Mackenzie Presbyterian Institute, 01302907, São Paulo, Brazil

*corresponding author: ali.nawaz@ieee.org; anawaz@fbk.eu

$\dagger$ in memoriam

Keywords: Defect-free P3HT, regioregularity, charge transport, space-charge-limited current, hole mobility, electron mobility

\section{Abstract}

The improvement of electron transport in polymer semiconductors is highly desirable for realizing robust, large-area and low-cost organic integrated circuits. This work investigates the effect of regioregularity on the intrinsic hole and electron transport characteristics of poly(3-hexylthiophene-2,5-diyl) (P3HT) using current-voltage $(I-V)$ measurements in metal/polymer/metal sandwich structures. Through a direct comparison between 93\% regioregular P3HT (EG-P3HT) and 100\% regioregular defect-free poly(3-hexylthiophene-2,5diyl) (DF-P3HT), it is found that the elimination of regioregularity defects improves the electron mobility of DF-P3HT $\left(1.05 \times 10^{-7} \mathrm{~cm}^{2} \mathrm{~V}^{-1} \mathrm{~s}^{-1}\right)$ by three orders of magnitude compared to the $93 \%$ regioregular EG-P3HT sample $\left(1.82 \times 10^{-10} \mathrm{~cm}^{2} \mathrm{~V}^{-1} \mathrm{~s}^{-1}\right)$. Quantum chemical calculations indicate that the improvement of electron mobility in DF-P3HT can be associated to the lower degree of disorder in these samples that tends to increase the transfer angle between the lowest unoccupied molecular orbitals of adjacent chains. At the same time, the lower dipole moments produced by the defect-free polymer molecules also appears to play an important role 
in decreasing the susceptibility of charge transport to environment-induced electron traps. The obtained results provide a strong evidence that the elimination of regioregularity defects is an effective technique to improve electron transport and restore the symmetry between hole and electron mobility in P3HT as well as other thiophene-based polymers.

\section{Introduction}

The field of organic semiconductors (OSCs) has shown immense progress over the past 50 years, moving from a topic of pure academic interest to the manufacture of low-cost industrial-scale devices. ${ }^{1,2}$ However, the problem of electron transport being much worse than hole transport in OSCs, especially in conjugated polymers (CPs), has been daunting scientists for as long as the emergence of this field. ${ }^{3,4}$ The main reason for poor electron transport characteristics in CPs is the presence of environment-induced electron traps that have empty orbitals below the lowest unoccupied molecular orbital (LUMO) level and can easily take up an electron hopping between neighboring LUMOs. Many applications in light-emitting diodes, photovoltaics, and thermoelectrics, etc. can benefit from better electron-transporting CPs. ${ }^{5}$ Similarly, CPs with comparable hole and electron transport performance are required by applications like ambipolar transistors and organic complementary circuits. ${ }^{6,7}$ Therefore, it is highly desirable to develop strategies that assist in improving electron transport in CPs.

Regioregular poly(3-hexylthiophene-2,5-diyl) (rr-P3HT) (Figure 1a) is one of the most commonly used CPs in organic electronic devices. ${ }^{8-11}$ This is mainly due to its ease of processability, high chemical stability, wide commercial availability, and ability to self-orient into a well-ordered lamellar structure. ${ }^{12,13}$ An important variant of P3HT is the $100 \%$ regioregular defect-free P3HT (DF-P3HT), in which the coupling defects along polymer chains are eliminated during the synthesis process, resulting in better crystallinity in polymer thin films. ${ }^{14}$ In our previous work, we have demonstrated that the application of defect-free P3HT (DF-P3HT) in organic field-effect transistors (OFETs) and photovoltaic devices results in remarkable improvement of overall device performance. ${ }^{15-18}$

Although it is well-known that the electron mobility of rr-P3HT is ca. 5 orders of magnitude lower than its hole mobility, ${ }^{19}$ the effect of the elimination of regioregularity defects on the intrinsic transport characteristics of rr-P3HT has still not been reported. In this work, we investigate the hole and electron transport of DF-P3HT using the space-charge-limited current (SCLC) model and provide a direct comparison with 93\% rr-P3HT (EG-P3HT). To facilitate the injection of positive charge carriers into the polymer films, we used a combination of indium-tin oxide (ITO) and poly(3,4-ethylenedioxythiophene):polystyrene sulfonate (PEDOT:PSS), whereas, the injection of negative charge carriers was made possible by using a combination of aluminum $(\mathrm{Al})$ and cesium oxide $\left(\mathrm{Cs}_{2} \mathrm{O}\right)$. Our results reveal that the electron mobility of rr-P3HT can be 
significantly improved when the regioregularity defects are completely eliminated through a specialized synthesis process. To understand the reason behind the huge difference in electron transport characteristics of non-100\% and $100 \%$ regioregular P3HT samples, we probe the structural and morphological properties of polymer thin films, and analyze the electrostatic aspects using quantum chemical calculations.

\section{Experimental}

For the preparation of the hole-transport devices, firstly the chemical etching of ITO (indium-tin oxide, Luminescence Technology Corp., $15 \Omega /$ sq) coated substrates was performed using zinc powder and an aqueous solution of hydrochloric acid (at a ratio $\mathrm{H}_{2} \mathrm{O}: \mathrm{HCl}$ of $2: 1$, v/v). ${ }^{20}$ Sequentially, the ITO substrates were cleaned in ultrasonic baths of acetone, deionized water (resistivity $>18.2 \mathrm{M} \Omega \mathrm{cm}^{-1}$ ), and isopropanol (20 min each) followed by annealing of the ITO films at $100{ }^{\circ} \mathrm{C}$ for $10 \mathrm{~min}$ to evaporate the residual solvent. The thickness of ITO film was measured as $100 \mathrm{~nm}$. PEDOT:PSS, Figure 1b and 1c, purchased from Sigma-Aldrich) was then spin-coated over glass/ITO substrates at $2000 \mathrm{rpm}$ for $60 \mathrm{~s}$ and sequentially annealed in a vacuum for 15 min at $100{ }^{\circ} \mathrm{C}$. The thickness of PEDOT:PSS film was measured as $20 \mathrm{~nm}$. The combination of ITO and PEDOT:PSS can be considered as an effective choice for injection of holes into P3HT because of the good match of energy levels (Figure 1d). DF-P3HT $\left(M_{n}: 41 \mathrm{kDa}\right)$ was synthesized via Nickel Zero Catalyzed Kumada Catalyst-Transfer Polymerization applying continuous flow synthesis, ${ }^{16}$ while EG-P3HT (93\% regioregular) was purchased from Luminescence Technology Corp. $\left(M_{n} \sim 27 \mathrm{kDa}\right)$. The P3HT films were prepared in toluene at concentrations of $15 \mathrm{mg} \mathrm{mL}^{-1}, 20 \mathrm{mg} \mathrm{mL}^{-1}, 25 \mathrm{mg} \mathrm{mL}^{-1}$, and $30 \mathrm{mg} \mathrm{mL}^{-1}$, and spincoated over PEDOT:PSS at $1500 \mathrm{rpm}$ for $60 \mathrm{~s}$, resulting in P3HT thicknesses of $120 \mathrm{~nm}, 200 \mathrm{~nm}, 300 \mathrm{~nm}$, and $380 \mathrm{~nm}$, respectively. After deposition, the P3HT films were annealed in a vacuum at $100{ }^{\circ} \mathrm{C}$ for $30 \mathrm{~min}$. For top contact, aluminum (Al) was evaporated at a base pressure of $\sim 10^{-6}$ Torr to form a $100 \mathrm{~nm}$ thin film. For the preparation of the electron-transport devices, glass substrates were cleaned by using the same procedure as described previously. The injection of electrons was obtained by using a combination of $\mathrm{Al}$ and $\mathrm{Cs}_{2} \mathrm{O}$ layers. $\mathrm{Cs}_{2} \mathrm{O}$ layer $(3 \mathrm{~nm})$ was formed over $\mathrm{Al}$ by thermal evaporation of cesium carbonate $\left(\mathrm{Cs}_{2} \mathrm{CO}_{3}\right.$, purchased from Sigma-Aldrich) at a rate of $0.1 \AA \mathrm{s}^{-1}$, which decomposed into $\mathrm{Cs}_{2} \mathrm{O}$. As reported previously, such a combination of $\mathrm{Al} / \mathrm{Cs}_{2} \mathrm{O}$ leads to work function reduction to $2.1 \mathrm{eV},{ }^{21}$ which guarantees effective injection of electrons-only into P3HT (LUMO $\approx 3 \mathrm{eV}$ ), see Figure 1e. In this case, the P3HT films were prepared in toluene at concentrations of $5 \mathrm{mg} \mathrm{mL}^{-1}, 8 \mathrm{mg} \mathrm{mL}^{-1}, 11 \mathrm{mg} \mathrm{mL}^{-1}$, and $14 \mathrm{mg} \mathrm{mL}^{-1}$, and spin-coated over $\mathrm{Al} / \mathrm{Cs}_{2} \mathrm{O}$ at 1500 rpm for $60 \mathrm{~s}$, resulting in P3HT thicknesses of $30 \mathrm{~nm}, 50 \mathrm{~nm}, 85 \mathrm{~nm}$, and $120 \mathrm{~nm}$, respectively. After deposition, the P3HT films were annealed in a vacuum at $100{ }^{\circ} \mathrm{C}$ for $30 \mathrm{~min}$. For top contact formation, $\mathrm{Cs}_{2} \mathrm{O}$ and $\mathrm{Al}$ were sequentially deposited using the procedure described earlier. 
The polymer film thicknesses were measured using a BrukerDektakXT surface profiler, while the absorption and morphological characteristics of rr-P3HT were analyzed using a Perkin Elmer LAMBDA 950 UV/Vis/NIR Spectrophotometer and a Shimadzu SPM-9500J3 scanning probe microscope, respectively. Xray Diffraction (XRD) data was obtained using a D8 Advance Bruker diffractometer in $\mathrm{SiO}_{2} / \mathrm{DF}-\mathrm{P} 3 \mathrm{HT}$

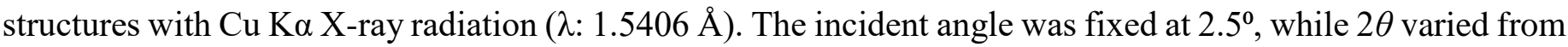
$3^{0}$ to $25^{\circ}$.

The quantum chemical calculations were performed by optimizing the geometries using density functional theory (DFT) with B3LYP, ${ }^{22} 3-21 \mathrm{G}^{23}$ and $6-31 \mathrm{G}(\mathrm{d}, \mathrm{p})^{24}$ basis set. The electronic structure, dipole moment and characteristic energies were obtained running single point calculations of the optimized structures at the same level of theory. The transfer integral $(t)$ calculations were carried out using the Gaussian 16 package. ${ }^{25}$ The $t$ between the LUMOs and the highest occupied molecular orbitals (HOMOs) of two optimized thiophene rings were obtained assuming a face-on (co-facial) configuration of two molecules separated by 4 $\AA$. In the initial configuration, $t$ was determined when the two rings match perfectly so that the overlap between the $\pi$-orbitals is maximum. Then a lateral displacement of $0.2 \AA$ was assumed in each step of $t$ calculations without changing the distance between the ring's planes using the same level of theory applied for the optimizations. The procedure was repeated until both integrals vanished to zero.

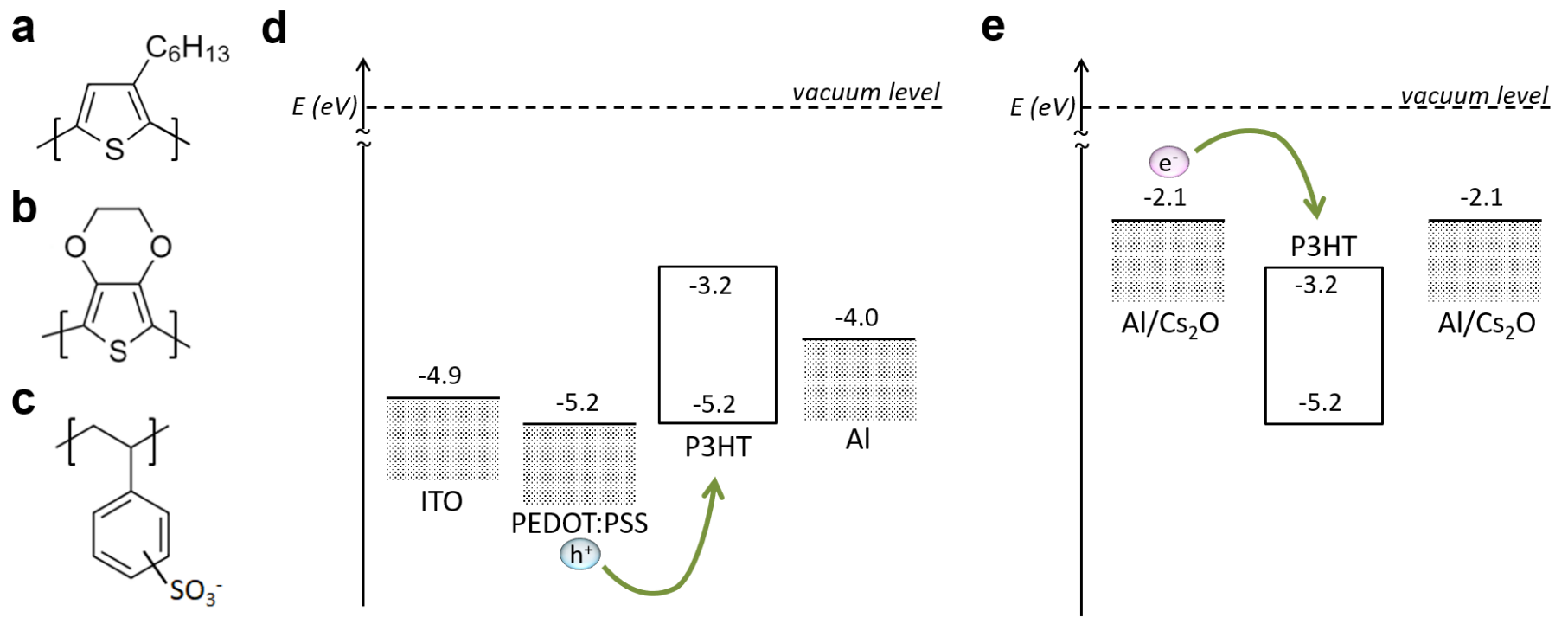

Figure 1 Chemical structures of (a) P3HT, (b) PEDOT, and (c) PSS. Energy level diagrams of (d) holetransporting P3HT diode structure, and (e) electron-transporting P3HT diode structure.

\section{Results and discussion}


The morphological and structural characteristics of DF-P3HT and EG-P3HT were investigated using atomic force microscopy (AFM), X-ray diffraction (XRD), and UV-vis absorbance spectroscopy. Figure 2a shows the AFM height image of the polymer films that exhibit isotropic nodule-like morphologies. The absence of regioregularity defects results in the formation of much bigger grains in DF-P3HT thin film compared to the EG-P3HT thin film. The structural order in the polymer films was investigated using out-of-plane XRD experiments (Figure $2 \mathrm{~b}$ ). The diffraction peak at $2 \theta \approx 5.5^{\circ}$ corresponds to the (100) alkyl chain direction. ${ }^{26}$ Using the Scherer formula, ${ }^{27}$ the crystallite diameter size of DF-P3HT and EG-P3HT was estimated to be $\sim 80$ $\AA$ and $\sim 30 \AA$, respectively, further asserting that DF-P3HT film consists of bigger crystallites. Figure 2c presents the absorbance spectra of the polymer films in the region of $\pi-\pi^{*}$ absorption. Comparatively, the DFP3HT data shows a more pronounced and intense $\mathrm{A}_{0-0}$ absorption peak, a red-shifted $\mathrm{A}_{0-1}$ peak, and a significantly less pronounced $\mathrm{A}_{0-2}$ absorption peak. These features imply improvement of both inter-and intrachain molecular order in the DF-P3HT film, and reduction in the number of disordered unaggregated molecules or segments with shorter conjugation lengths. ${ }^{28-30}$
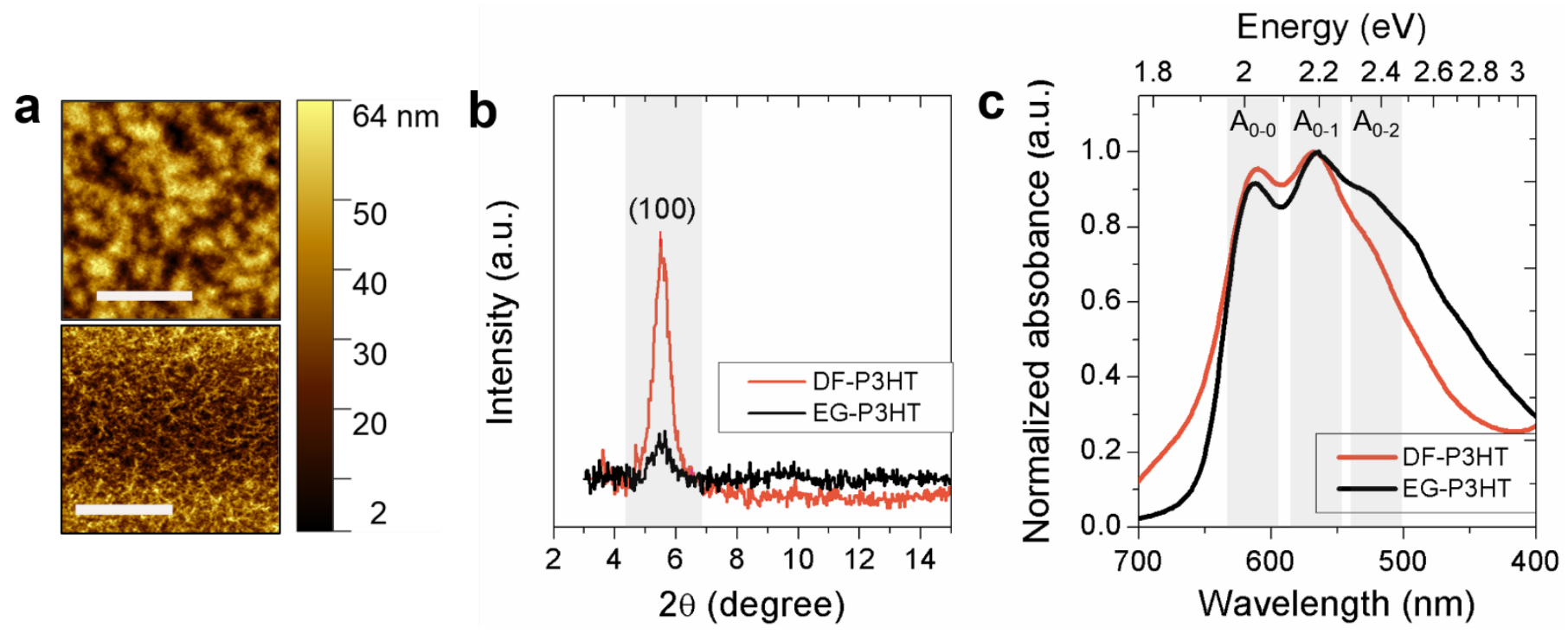

Figure 2 (a) AFM image of DF-P3HT (top panel; Ra = $11.74 \mathrm{~nm}$ ) and EG-P3HT (bottom panel; Ra = 6.63 $\mathrm{nm}$ ) films deposited on glass. Scale bar corresponds to $4 \mu \mathrm{m}$ in both cases. (b) XRD patterns of DF-P3HT and EG-P3HT thin films. (c) UV-vis absorbance spectra of DF-P3HT and EG-P3HT thin films.

The intrinsic hole and electron mobilities of DF-P3HT and EG-P3HT were extracted electrically using the SCLC method ( $I-V$ characteristics shown in Figure $3 \mathrm{a}$ and $3 \mathrm{~b}$ ). In the case of hole injection, ITO/PEDOT:PSS was positively biased, whereas, for electron injection, $\mathrm{Al} / \mathrm{Cs}_{2} \mathrm{O}$ was negatively biased. At low voltages, the $I-V$ characteristics follow Ohm's law, with $\partial \log \mathrm{I} / \partial \log \mathrm{V} \approx 1$. The current density $(j)$ in this region can be written as ${ }^{31}$ : 


$$
j=q \mu n_{0} \frac{V}{d}
$$

where, $q$ is the electronic charge, $\mu$ is the charge-carrier mobility, $V$ is the applied voltage, $d$ is the thickness of the polymer film, and $n_{0}$ is the free carrier density, given by:

$$
n_{0}=\frac{9}{8} \frac{\varepsilon V_{\Omega}}{q d^{2}}
$$

where, $\varepsilon$ is the polymer's permittivity, and $V_{\Omega}$ corresponds to the transition voltage between the ohmic and the SCLC region. Upon application of sufficient bias, accumulated space charge starts to limit the transport of charge carriers through the polymer film, and the SCLC is described by Mott-Gurney law ${ }^{32,33}$ :

$$
j=\frac{9}{8} \varepsilon \mu \frac{V^{2}}{d^{3}}
$$

The calculated hole mobility of DF-P3HT in the SCLC regime was found to be slightly higher than EG-P3HT, whereas, the electron mobility of DF-P3HT showed significant improvement by three orders of magnitude (Table 1). Figure 3c shows the dependence of hole and electron currents on DF-P3HT film thickness. In SCLC theory, it is expected that $(\partial \log I / \partial \log d)=-3$ when the $I$ values are taken at a constant $V$, as also evident from Equation 3. In our case, we have obtained $\partial \log I / \partial \log d=-2.98$ for hole currents at $\mathrm{V}=-2 \mathrm{~V}$, $\operatorname{and} \partial \log I / \partial \log d=$ -2.27 for electron currents at $\mathrm{V}=4 \mathrm{~V}$ for four different DF-P3HT film thicknesses. The observed thickness dependence, with the derivative near to 3, further confirms SCLC transport in the P3HT films.
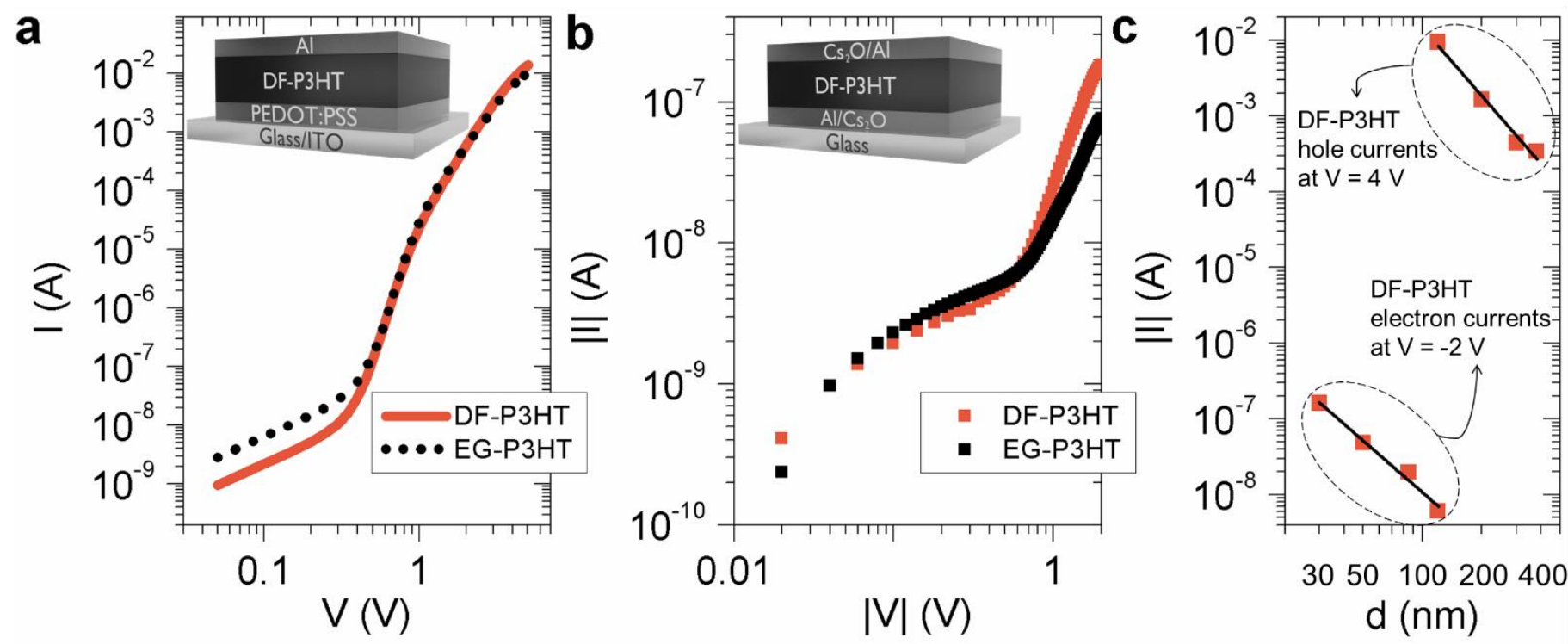

Figure 3 Typical $I-V$ characteristics of DF-P3HT and EG-P3HT devices (in log-log scale) for calculation of (a) hole mobility (ITO/PEDOT:PSS positively biased) and (b) electron mobility ( $\mathrm{Al} / \mathrm{Cs}_{2} \mathrm{O}$ negatively biased). Upper left insets show the corresponding device structures. (c) Thickness dependence of hole and electron currents of DF-P3HT in the SCLC regime. For hole currents, 120, 200, 300, and $380 \mathrm{~nm}$ film thicknesses were 
considered, while for electron currents 30, 50, 85, and $120 \mathrm{~nm}$ film thicknesses were considered. The solid black lines correspond to theoretical fits with $\partial \log I / \partial \log d=-2.98$ for hole currents and $\partial \log I / \partial \log d=-2.27$ for electron currents.

Table 1 Summary of hole and electron mobilities of DF- and EG-P3HT.

\begin{tabular}{lcc}
\hline Sample & $\begin{array}{c}\text { Hole mobility } \\
\left(\mathbf{c m}^{\mathbf{2}} \mathbf{V}^{-\mathbf{1}} \mathbf{s}^{\mathbf{- 1}}\right)\end{array}$ & $\begin{array}{c}\text { Electron mobility } \\
\left(\mathbf{c m}^{\mathbf{2}} \mathbf{V}^{\mathbf{- 1}} \mathbf{s}^{\mathbf{- 1}}\right)\end{array}$ \\
\hline DF-P3HT & $3.29 \times 10^{-4}$ & $1.05 \times 10^{-7}$ \\
EG-P3HT & $1.57 \times 10^{-4}$ & $1.82 \times 10^{-10}$ \\
\hline
\end{tabular}

The overall improvement in the intrinsic mobility of DF-P3HT may be attributed to the complete elimination of synthesis-induced regioregularity defects, which increases the $\pi$-conjugation length of $\mathrm{P}_{3} \mathrm{HT}^{14}$ and improves its overall morphological and structural order, as observed in Figure 2. Yet the electron mobility increases by three orders of magnitude compared to only a slight increase in the hole mobility. In organic systems the hopping mobility tends to decrease for lower values of $t$ between the frontier orbitals. One reason behind the lower electron mobility compared to the hole mobility can be attributed to the smaller value of $t$ for LUMOs $\left(t_{L U M O}\right)$ in comparison to that for HOMOs $\left(t_{\text {HOMO }}\right)$. In this case, it is instructive to consider the data in Figure 4a, which shows the variation of the transfer integrals between two co-facial thiophene rings as they are laterally displaced. Although the system involving two thiophenes is much simpler than the interaction between P3HT chains, it produced useful insights that can assist in understanding our experimental results. It can be seen that $t_{L U M O}<t_{\text {HOMO }}$ for the perfect co-facial configuration when the overlap between the $\pi$-orbitals is the highest (Figure 4a). This result suggests that the hole mobility is indeed intrinsically higher than the electron mobility in this system. However, the key result in Figure $4 \mathrm{a}$ is that the $t_{L U M O}$ is much more susceptible to

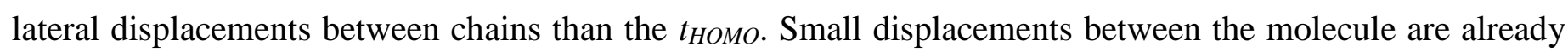
able to collapse $t_{L U M O}$ to zero. This does not happen for $t_{H O M O}$, which is more resilient to variations in the configuration and starts to oscillate with a decreasing amplitude as the displacement increases. This result indicates that reduction of small mismatching between adjacent P3HT chains (induced by defects in the regioregularity) tends to have a stronger impact on the electron mobility than on the hole mobility. This may explain why the electron mobility in the DF-P3HT system is significantly higher than the electron mobility in the EG-P3HT system.

To further investigate the influence of regioregularity defects on the electronic environment, we calculated the dipole moments of P3HT molecules using quantum chemical calculations. In this case, we considered two sets of P3HT oligomers with 9 repeat units. One set consists of a non-100\% regioregular structure with a TT-HT regioregularity defect, while the other set consists of a defect-free regioregular structure 
with $100 \%$ head-tail (HT) coupling. The optimized structures of the non-100\% and 100\% regioregular configurations are shown in Figure 4b. In non-100\% regioregular P3HT, the large torsional angle at the central thiophene ring $\left(68.65^{\circ}\right)$ practically divides the chains in two segments with different planes of orientation. This distortion partially breaks the $\pi$-conjugation of the chain, decreasing the delocalization of the frontier orbitals. Similarly, the high torsional angle between adjacent thiophene rings results in a non-planar backbone, resulting in a high dipole moment $(\eta(\mathrm{D}))$ per thiophene ring (0.107) (Table 2). The larger density of stronger dipole moments produced by the non-100\% regioregular structure can hinder molecular ordering and large crystallite formation, as indicated by our experimental results (Figure 2). On the other hand, in the 100\% regioregular P3HT structure, the torsional angle between adjacent thiophene rings fluctuates smoothly around $30^{\circ}$. The high planarity of the $100 \%$ regioregular P3HT chains results in high delocalization of the frontier orbitals, producing a lower $\eta(\mathrm{D})$ per thiophene ring (0.053) (Table 2). The lower torsional angles between adjacent thiophene rings would result in improved $\pi$-conjugation lengths, close inter-molecular packing, and bigger grain and crystallite formation, as indicated by our experimental results (Figure 2).

Table 2 Torsional angle between neighboring thiophene rings, and dipole moment magnitude per thiophene ring of P3HT oligomers with 9 repeat units as obtained by DFT method at the B3LYP/3$21 \mathrm{G}$ level of theory.

\begin{tabular}{ccc}
\hline $\begin{array}{c}\text { P3HT } \\
\text { structure }\end{array}$ & Torsional angle & $\boldsymbol{\eta}(\mathbf{D})$ \\
\hline $\begin{array}{c}100 \% \\
\text { regioregular } \\
\text { Non-100\% } \\
\text { regioregular }\end{array}$ & $30^{\circ}$ & 0.053 \\
\hline
\end{tabular}

The difference in dipole moments of non-100\% and 100\% regioregular P3HT may help in further understanding the improvement of DF-P3HT electron mobility. It has been previously reported that semiconducting polymers typically show lower electron mobilities in air because the adsorbed water and oxygen molecules form oxygen-water complexes at an energy level of around $-3.6 \mathrm{eV}$ with respect to vacuum, with a typical distribution width of $\sim 0.1 \mathrm{eV} .{ }^{3}$ Since the LUMO of most polymers ranges from around -2 to -3 $\mathrm{eV}$, these hydrated oxygen complexes act as traps for electrons that reduce the mean mobility of the negative charge carriers. This explains why the electron mobility of P3HT is significantly lower than its hole mobility. However, the relatively higher electron mobility in DF-P3HT compared to EG-P3HT (Table 1) means that electron transport in DF-P3HT is relatively less susceptible to the oxygen-water related electron traps. This 
can be understood by the lower dipole moments produced by the $100 \%$ regioregular P3HT molecules (Table 2), whose polarization energy is less likely to be modified by the dipole moments produced by the oxygenwater complexes. On the other hand, the more polar non-100\% regioregular P3HT molecules are expected to interact strongly with the dipole moments produced by the oxygen-water complexes, which may modify the polarization energy of neighboring P3HT molecules, resulting in the localization of otherwise mobile charge carriers and thus leading to a lower mobility.
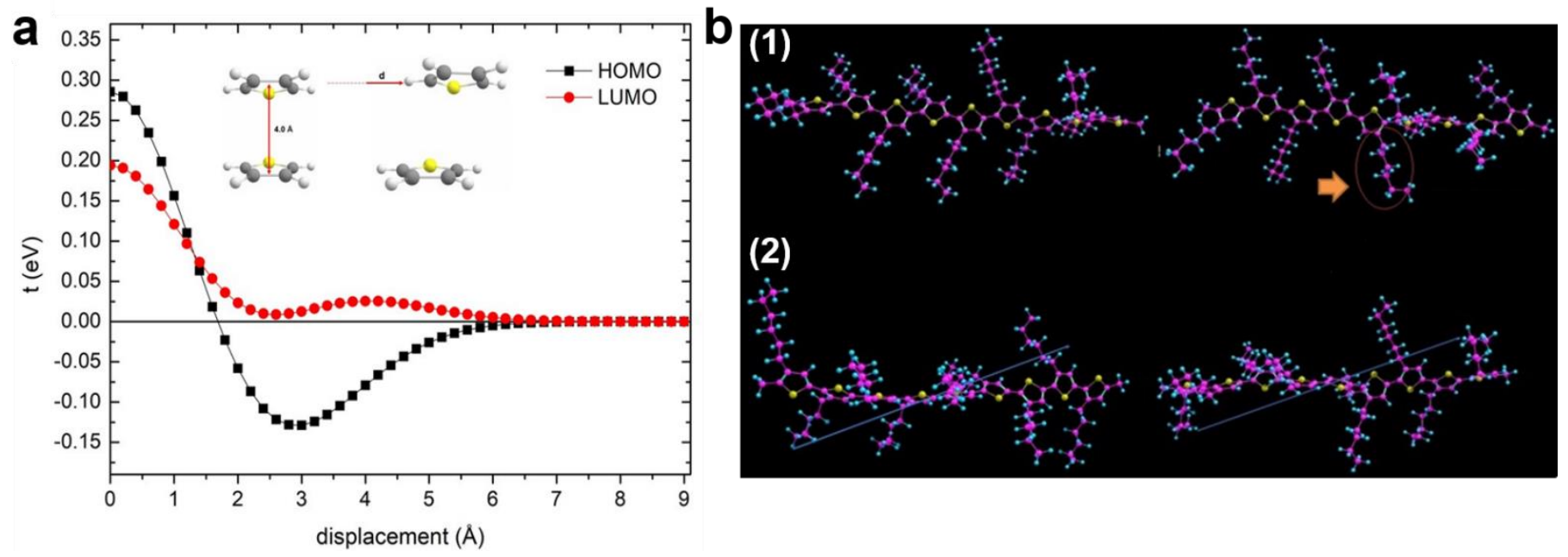

Figure 4 (a) Variation of $t$ for the HOMO and LUMO of two thiophene rings with the lateral displacement between them. Zero corresponds to the perfect co-facial configuration between the rings separated by $4 \AA$. The molecules were laterally displaced by $0.2 \AA$ at each step. The values of $t$ were obtained using DFT/B3LYP/6$31 \mathrm{G}(\mathrm{d}, \mathrm{p})$ level of theory (details in the experimental section). The inset illustrates the procedure followed to calculate $t$. (b1) Optimized structure of the 9-units P3HT oligomer with a 100\% regioregular configuration (left) and with a non-100\% regioregular configuration (right). (b2) The orientation of the dipole moment vector in the negative to positive charge convention.

Our theoretical results clearly indicate that the favorable electrostatic environment induced by the elimination of P3HT's regioregularity defects could be playing an important role in improving its electronic mobility. In particular, it appears that two mechanisms are acting simultaneously to improve the electron mobility of DF-P3HT compared to EG-P3HT. Firstly, the lower degree of disorder in the DF-P3HT system tends to increase the transfer angle between the LUMOs of adjacent chains, and secondly the lower dipole moments produced by the DF-P3HT molecules makes them less susceptible to the oxygen-water related electron traps induced by the environment. The higher electron mobility observed in the case of DF-P3HT further suggests that there may exist a direct correlation between P3HT regioregularity and electron transport. The experiments performed in our laboratory also show an almost linear increase in electron mobility with an 
increase in the regioregularity of P3HT from 93\% to 100\%. This trend is illustrated in Figure 5, which also shows a comparison of mobilities observed in this work with other P3HT reports ${ }^{19,34-36}$ as well as with several other reports on classical and emerging polymer materials. ${ }^{37-46}$ The hole mobility of DF-P3HT is comparable or higher than that of non-100\% rr-P3HT ${ }^{19,36}$ and other high performance polymer materials like poly2methoxy-5-(2-ethylhexyloxy-1,4-phenylenevinylene) (MEH-PPV), ${ }^{40}$ PTZ6 (polymer based on alkoxylfluorophenyl substituted benzodithiophene), ${ }^{42}$ TBFPS-BT (polymer based on asymmetrical thieno[2,3f]benzofuran building block), ${ }^{43}$ and a dithienopyrrolobenzothiadiazole (DTPBT)-based polymer. ${ }^{44}$ The noteworthy improvement, however, is observed in the case of the electron mobility of DF-P3HT, which is three orders of magnitude higher than that of $93 \%$ rr-P3HT and two orders of magnitude higher than that of $98 \%$ rrP3HT. ${ }^{19}$ Our work offers a new way of improving electron mobility in thiophene-based regioregular polymers ${ }^{47}$ i.e. by complete elimination of synthesis-induced regioregularity defects. This effective technique can hence be used to restore symmetry between hole and electron transport in thiophene-based materials, which would have obvious benefits in complementary electronic applications based on polymers ${ }^{48,49}$ as well as smallmolecules. ${ }^{50}$ Therefore, our work sheds light on a key structure-function relationship of P3HT, which can have both fundamental scientific as well as practical implications.

The successful measurement of electron mobility using $I-V$ measurements is another important aspect of our work, which was made possible by using $\mathrm{Al} / \mathrm{Cs}_{2} \mathrm{O}$ as the electron injecting electrode. The preparation of efficient contacts for negative charge carriers is a difficult task, and low work function materials like calcium and barium ${ }^{51}$ are usually highly reactive to oxygen and moisture that affects device stability. ${ }^{21}$ In this context, the use of an electron injection layer, such as $\mathrm{Cs}_{2} \mathrm{O}$, between the cathode and the electron transport layer is an effective strategy since it not only decreases the electron injection barrier but also avoids the need for employing highly reactive metals. Hence, our work further highlights the effectiveness of $\mathrm{Cs}_{2} \mathrm{O}$ as the electron injection layer in electronic devices. ${ }^{52,53}$ 


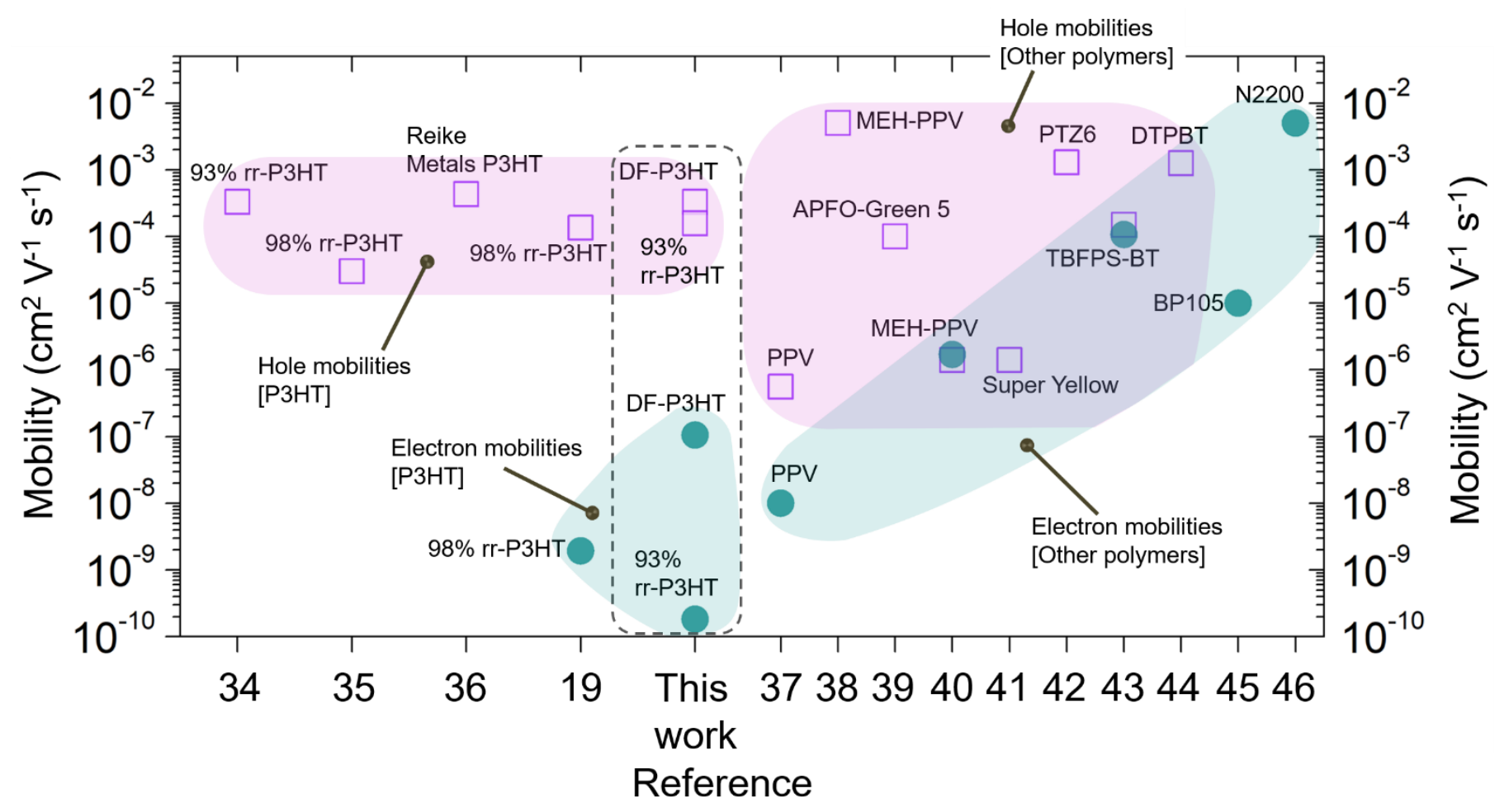

Figure 5 Comparison of hole and electron mobilities obtained in this work (points enclosed within the dashed rectangle) with mobilities of various polymer semiconductors reported in the literature.

\section{Conclusions}

Our experimental results provide a strong correlation between regioregularity and electron transport. We have calculated the electron and hole mobilities in P3HT using $I$ - $V$ measurements in simple sandwich-like device structures. By fitting the resulting data to the SCLC model, we determined hole and electron mobility of $3.29 \times 10^{-4} \mathrm{~cm}^{2} \mathrm{~V}^{-1} \mathrm{~s}^{-1}$ and $1.05 \times 10^{-7} \mathrm{~cm}^{2} \mathrm{~V}^{-1} \mathrm{~s}^{-1}$, respectively for $100 \%$ regioregular DF-P3HT, and $1.57 \times$ $10^{-4} \mathrm{~cm}^{2} \mathrm{~V}^{-1} \mathrm{~s}^{-1}$ and $1.82 \times 10^{-10} \mathrm{~cm}^{2} \mathrm{~V}^{-1} \mathrm{~s}^{-1}$, respectively for $93 \%$ regioregular EG-P3HT. Our quantum chemical calculation results suggest that two key mechanisms could be largely influencing the remarkable improvement of electron mobility in DF-P3HT; 1) increase in transfer angle between the LUMOs of adjacent chains, and 2) decrease in the dipole moments produced by the defect-free molecules, which can make the charge transport less susceptible to the oxygen-water related electron traps. Our findings clearly indicate that the elimination of regioregularity defects may be seen as an effective method to improve the electron mobility of P3HT, and possibly of other thiophene-based regioregular polymers. More importantly, our results pave the way for new research directions that focus on comprehensively investigating the dependence of electron-transport on regioregularity. 


\section{Conflicts of interest}

There are no conflicts to declare.

\section{Acknowledgements}

DST India and CNPq-Brazil are acknowledged for bilateral funding. Ali Nawaz, Anne B. R. Abreu, Marlus Koehler, Jose P. M. Serbena, and Ivo A. Hümmelgen acknowledge CNPq-Brazil. Leandro Merces gratefully acknowledges FAPESP (process 18/18136-0). Centre for Electronic Microscopy (CME-UFPR) is acknowledged for AFM and XRD experiments. Anil Kumar acknowledges Department of Information Technology (DIT) India for funding.

\section{References}

1 W. Brütting, Ed., Physics of Organic Semiconductors, Wiley, 2005.

2 A. Köhler and H. Bassler, Electronic Processes in organic semiconductors, Wiley-VCH, 2015.

3 H. T. Nicolai, M. Kuik, G. A. H. Wetzelaer, B. de Boer, C. Campbell, C. Risko, J. L. Brédas and P. W. M. Blom, Nat. Mater., 2012, 11, 882-887.

4 A. Köhler, Nat. Mater., 2012, 11, 836-837.

5 H. Klauk, Organic electronics: materials, manufacturing, and applications, Wiley-VCH, 2006.

6 J. Zaumseil and H. Sirringhaus, Chem. Rev., 2007, 107, 1296-1323.

7 G. Gelinck, P. Heremans, K. Nomoto and T. D. Anthopoulos, Adv. Mater., 2010, 22, 3778-3798.

8 C. De Col, A. Nawaz, I. Cruz-Cruz, A. Kumar, A. Kumar and I. A. Hümmelgen, Org. Electron., 2015, 17, 22-27.

9 I. A. Hümmelgen, J. Solid State Electrochem., 2017, 21, 1977-1985.

10 L. Zheng, X. Deng, Y. Wang, J. Chen, X. Fang, L. Wang, X. Shi and H. Zheng, Adv. Funct. Mater., 2020, 30, 2001604.

11 L. Salamandra, L. La Notte, C. Fazolo, M. Di Natali, S. Penna, L. Mattiello, L. Cinà, R. Del Duca and A. Reale, Org. Electron., 2020, 81, 105666.

12 Z. Bao, A. Dodabalapur and A. J. Lovinger, Appl. Phys. Lett., 1996, 69, 4108-4110.

13 H. Sirringhaus, P. J. Brown, R. H. Friend, M. M. Nielsen, K. Bechgaard, B. M. W. Langeveld-Voss, A. J. H. Spiering, R. A. J. Janssen, E. W. Meijer, P. Herwig and D. M. de Leeuw, Nature, 1999, 401, 685-688.

14 P. Kohn, S. Huettner, H. Komber, V. Senkovskyy, R. Tkachov, A. Kiriy, R. H. Friend, U. Steiner, W. T. S. Huck, J.-U. Sommer and M. Sommer, J. Am. Chem. Soc., 2012, 134, 4790-4805.

15 N. Chandrasekaran, E. Gann, N. Jain, A. Kumar, S. Gopinathan, A. Sadhanala, R. H. Friend, A. Kumar, C. R. McNeill and D. Kabra, ACS Appl. Mater. Interfaces, 2016, 8, 20243-20250. 
16 A. Nawaz, M. S. Meruvia, D. L. Tarange, S. P. Gopinathan, A. Kumar, A. Kumar, H. Bhunia, A. J. Pal and I. A. Hümmelgen, Org. Electron., 2016, 38, 89-96.

17 A. Nawaz, A. Kumar and I. A. Hümmelgen, Org. Electron., 2017, 51, 94-102.

18 A. Nawaz, I. Cruz-Cruz, J. S. Rego, M. Koehler, S. P. Gopinathan, A. Kumar and I. A. Hümmelgen, Semicond. Sci. Technol., 2017, 32, 084003.

19 M. F. Ahmed and I. A. Hummelgen, Int. J. Electroact. Mater., 2013, 1, 60-63.

20 G. Bradshaw and A. J. Hughes, Thin Solid Films, 1976, 33, L5-L8.

21 P. Tyagi, R. Srivastava, A. Kumar, S. Tuli and M. N. Kamalasanan, Org. Electron., 2013, 14, 13911395.

22 A. D. Becke, J. Chem. Phys., 1993, 98, 5648-5652.

23 M. J. Frisch, G. W. Trucks, H. B. Schlegel, G. E. Scuseria, M. A. Robb, J. R. Cheeseman, J. A. Montgomery Jr, T. Vreven, K. N. Kudin, J. C. Burant and J. M. Millam, Gaussian 03, Revision C. 02, Wallingford, CT: Gaussian. Inc., 2004.

24 Z. Zheng, J.-L. Brédas and V. Coropceanu, J. Phys. Chem. Lett., 2016, 7, 2616-2621.

25 M. J. Frisch, G. W. Trucks, H. B. Schlegel, G. E. Scuseria, M. A. Robb, J. R. Cheeseman, G. Scalmani, V. Barone, B. Mennucci and G. A. Petersson, Gaussian 16, Revision C.01, Gaussian, Inc., Wallingford CT, 2016.

26 R. J. Kline, M. D. McGehee, E. N. Kadnikova, J. Liu and J. M. J. Fréchet, Adv. Mater., 2003, 15, 1519-1522.

27 A. L. Patterson, Phys. Rev., 1939, 56, 978-982.

28 P. J. Brown, D. S. Thomas, A. Köhler, J. S. Wilson, J.-S. Kim, C. M. Ramsdale, H. Sirringhaus and R. H. Friend, Phys. Rev. B, 2003, 67, 064203.

29 F. C. Spano, J. Chem. Phys., 2005, 122, 234701.

30 J. Clark, C. Silva, R. H. Friend and F. C. Spano, Phys. Rev. Lett., 2007, 98, 206406.

31 M. A. Lampert, Phys. Rev., 1956, 103, 1648-1656.

32 M. A. Lampert and P. Mark, Current Injection in Solids, Academic Press, New York, 1981.

33 K. C. Kao and W. Hwang, Electrical Transport in Solids, Pergamon, Oxford, 1981.

34 C. Goh, R. J. Kline, M. D. McGehee, E. N. Kadnikova and J. M. J. Fréchet, Appl. Phys. Lett., 2005, 86, 122110.

35 Z. Chiguvare and V. Dyakonov, Phys. Rev. B, 2004, 70, 235207.

36 H. K. H. Lee, K. K. H. Chan and S. K. So, Org. Electron., 2012, 13, 541-544.

37 H. C. F. Martens, J. N. Huiberts and P. W. M. Blom, Appl. Phys. Lett., 2000, 77, 1852.

38 H.-F. Meng, B.-L. Chen, K.-C. Tzeng and S.-F. Horng, Appl. Phys. Lett., 2006, 88, 023505.

39 L. Mattias Andersson, W. Osikowicz, F. L. E. Jakobsson, M. Berggren, L. Lindgren, M. R. Andersson 
and O. Inganäs, Org. Electron., 2008, 9, 569-574.

40 Y. Zhang, B. de Boer and P. W. M. Blom, Phys. Rev. B, 2010, 81, 085201.

41 S. Gambino, A. K. Bansal and I. D. W. Samuel, Org. Electron., 2010, 11, 467-471.

42 B. Guo, W. Li, X. Guo, X. Meng, W. Ma, M. Zhang and Y. Li, Nano Energy, 2017, 34, 556-561.

43 D. He, L. Qiu, J. Yuan, Z.-G. Zhang, Y. Li and Y. Zou, Polymer (Guildf)., 2017, 114, 348-354.

44 V. J. Bhanvadia, H. K. Machhi, S. S. Soni, S. S. Zade and A. L. Patel, Polymer (Guildf)., 2020, 211, 123089.

45 C.-M. Yang, H.-H. Liao, S.-F. Horng, H.-F. Meng, S.-R. Tseng and C.-S. Hsu, Synth. Met., 2008, 158, $25-28$.

46 R. Steyrleuthner, M. Schubert, F. Jaiser, J. C. Blakesley, Z. Chen, A. Facchetti and D. Neher, Adv. Mater., 2010, 22, 2799-2803.

47 B. Amna, H. M. Siddiqi, A. Hassan and T. Ozturk, RSC Adv., 2020, 10, $4322-4396$.

48 Z. Ni, H. Wang, Q. Zhao, J. Zhang, Z. Wei, H. Dong and W. Hu, Adv. Mater., 2019, 31, 1806010.

49 G. L. Pozzoli, L. Merces, E. Yassitepe, V. B. de Morais, D. H. S. de Camargo and C. C. B. Bufon, ACS Appl. Nano Mater., 2020, 3, 3060-3070.

50 L. M. M. Ferro, L. Merces, D. H. S. Camargo and C. C. B. Bufon, Adv. Mater., 2021, 2101518.

51 S. Stolz, M. Petzoldt, S. Dück, M. Sendner, U. H. F. Bunz, U. Lemmer, M. Hamburger and G. Hernandez-Sosa, ACS Appl. Mater. Interfaces, 2016, 8, 12959-12967.

52 A. Hoff, I. Cruz-Cruz, M. C. Siqueira, K. D. Machado and I. A. Hümmelgen, Sol. Energy Mater. Sol. Cells, 2017, 171, 1-7.

53 S. Wang, H. Zhang, B. Zhang, Z. Xie and W.-Y. Wong, Mater. Sci. Eng. R Reports, 2020, 140, 100547. 